\title{
Acumulación de metales pesados en suelo y plantas de cuatro cultivos hortícolas, regados con agua del río Bogotá
}

\section{Accumulation of heavy metals in soil and plants of four vegetable crops irrigated with water of Bogota river}

\section{DIEGO MIRANDA ${ }^{1,2}$ \\ CARLOS CARRANZA ${ }^{1}$ \\ CARLOS ANDRÉS ROJAS \\ CARLOS MARTÍN JEREZ1 \\ GERHARD FISCHER ${ }^{1}$ \\ JORGE ZURITA'}

Planta de lechuga, cultivada en la cuenca media del río Bogotá.

Foto: C. Carranza

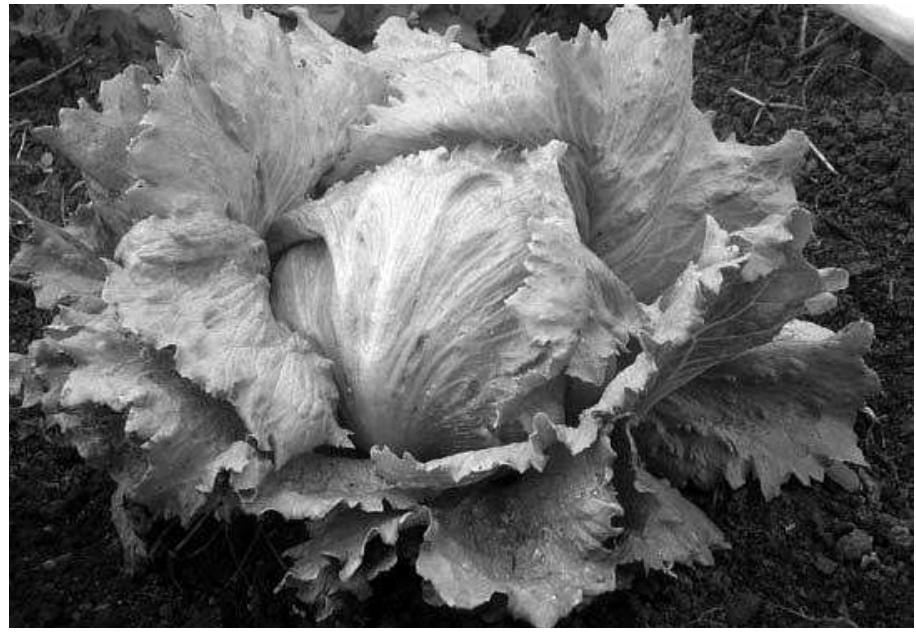

\section{RESUMEN}

En los cultivos hortícolas establecidos en la Sabana de Bogotá los productores desarrollan actividades de riego utilizando las aguas del río Bogotá a través del distrito de riego La Ramada, que cubre unas 6.400 ha. Su uso ha generado una problemática de contaminación en las especies hortícolas causando deterioro de la calidad de los productos que en su mayoría son consumidos en fresco. El presente estudio se desarrolló en plantaciones de lechuga ('Coolguard'), apio ('Tall Utah'), repollo (híbrido Delus) y brócoli (híbrido Legacy) en una finca en Soacha (vereda Canoas), Cundinamarca, ubicada en la cuenca media del río Bogotá, analizando los niveles de metales pesados plomo $(\mathrm{Pb})$, cadmio $(\mathrm{Cd})$, arsénico (As) y mercurio $(\mathrm{Hg})$ en agua, suelo y en la parte comestible de las plantas. Se encontró que las concentraciones de $\mathrm{Cd}$ en el agua del riego estuvieron cercanas a los límites establecidos por las normas vigentes nacionales e internacionales, mientras que los niveles en el suelo de las dos plantaciones estuvieron dentro del rango normal. La lechuga y el apio, a los 74 días después transplante, con 0,40 y $0,43 \mathrm{mg} \mathrm{kg}^{-1}$ peso fresco, respectivamente, presentaron contaminación con Cd superando el límite de la norma de la Unión Europea. Se discuten las posibles razones agroecológicas, fisiológicas y de muestreo para este comportamiento. En las cuatro hortalizas, la concentración de $\mathrm{Pb}$ superó la concentración máxima permitida en alimentos para lactantes y niños de corta edad establecida por la Unión Europea. En general, la lechuga acumuló niveles más altos de metales pesados que las otras tres especies.

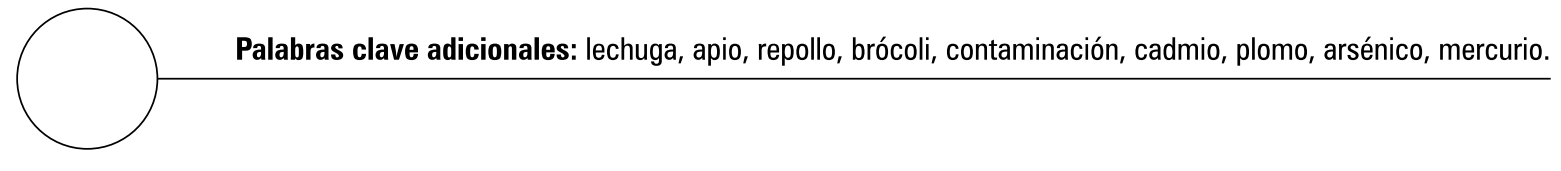

1 Departamento de Agronomía, Facultad de Agronomía, Universidad Nacional de Colombia, Bogotá (Colombia).

2 Autor para correspondencia.dmirandal@unal.edu.co 


\section{ABSTRACT}

In horticultural crops established in the Colombian Bogota Plateau, farmers develop activities of irrigation utilizing water of the Bogota river through the Irrigation District "La Ramada" which is covering 6,400 ha of land. This water use generated a problem of contamination in these crops causing quality deterioration of the produce, which in mainly consumed freshly. The present study was carried out in plantations of lettuce ('Coolguard') celery ('Tall Utah'), cabbage (Delus hybrid) and broccoli (Legacy hybrid) in a farm in Soacha (Canoas vereda), Cundinamarca province. The farm is located in the middle part of the Bogota river and the heavy metals lead $(\mathrm{Pb})$, cadmium $(\mathrm{Cd})$, arsenic $(\mathrm{As})$ and mercury $(\mathrm{Hg})$ in water, soil and edible part of plants were analyzed. It was found that concentrations of $\mathrm{Cd}$ in the irrigation water were near the limits established by national and international legal standards. Heavy metal levels of the soil in the two plantations were in the normal range. Lettuce and celery plants, at 74 days after transplanting, with 0.40 and $0.43 \mathrm{mg} \mathrm{kg}{ }^{-1}$ fresh weight, respectively, were contaminated with Cd exceeding the limits of the European Union norms. Possible agroecological, physiological and sampling reasons for this behavior are discussed. In the four vegetables lead contents exceeded the maximum allowed concentration in foods for children still on milk-feeded and young children, established by the European Union. En general, lettuce plants accumulated higher levels of heavy metals than the other three vegetable species.

Additional key words: lettuce, celery, cabbage, broccoli, contamination, heavy metals, cadmium, lead, arsenic, mercury.

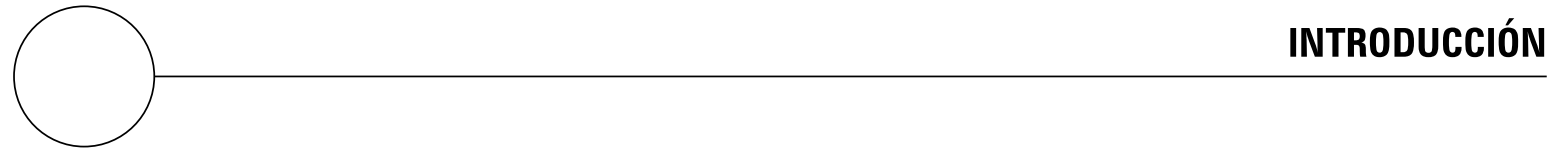

La cuenca del río Bogotá tiene una de las estructuras económicas más diversificadas en el país. Está estrechamente relacionada con la utilización de los recursos naturales por medio de actividades como agricultura, minería y transformación industrial (Secretaría de Agricultura de Cundinamarca, 2004). La cuenca del río Bogotá se divide en tres tramos, el primero, la cuenca alta que se encuentra en la zona norte de la ciudad de Bogotá, la cuenca media, y la cuenca baja que es la que recibe las aguas residuales de la misma ciudad y las de algunos municipios de la zona sur hasta su desembocadura en el río Magdalena (EAAB, 2002). La cuenca media del río Bogotá es la principal fuente de agua para la producción agrícola en los municipios aledaños del noroccidente y sur de la ciudad de Bogotá, que comprende 650 ha de cultivos hortícolas.

Los vertimientos a los que se ve sometido el río Bogotá a lo largo de su recorrido generan proble- mas de salud en la población, tanto en la margen oriental como occidental de su cauce, pero el problema más grave se genera sobre la población que habita la margen oriental del río, principalmente por la producción de hortalizas desarrollada en la localidad de Bosa y el municipio de Soacha (EAAB, 2002).

La contaminación del río Bogotá por los vertimientos de aguas residuales de los municipios de la cuenca ha sido un problema a resolver desde la primera mitad del siglo anterior. En su recorrido drena las aguas de $6.000 \mathrm{~km} 2$, donde habitan alrededor de 8,04 millones de habitantes en 42 municipios, incluyendo el Distrito Capital (CONPES, 2004). Han sido muchos los estudios realizados por diferentes investigadores y entidades (por ejemplo: CAR, 1991; González y Mejía, 1995; Vargas y Mejía, 1996; Montenegro,_2000) en los que se ha demostrado el alto grado de contaminación del río, con elementos traza como el 
arsénico (As), cadmio (Cd), cobalto (Co), cromo $(\mathrm{Cr})$, cobre $(\mathrm{Cu})$, hierro $(\mathrm{Fe})$, mercurio $(\mathrm{Hg})$, manganeso $(\mathrm{Mn})$, níquel $(\mathrm{Ni})$, plomo $(\mathrm{Pb})$, estaño $(\mathrm{Sn})$, cinc $(\mathrm{Zn})$, y una amplia variedad de sustancias orgánicas e inorgánicas potencialmente tóxicas.

En el distrito de riego La Ramada que cubre un área bruta de 6.400 ha, los suelos están desarrollados principalmente sobre sedimentos aluviales finos del valle del río Bogotá en un 75\% del área aproximadamente. En comparación con el tipo de suelo que predomina en la Sabana de Bogotá, estos suelos aluviales poseen texturas arcillosas pesadas, nivel freático fluctuante y drenaje natural pobre (CPT, 1985).

La producción de hortalizas en Colombia, en términos generales, es una actividad riesgosa que se caracteriza por los siguientes aspectos: cultivos de tamaño pequeño (0,5-10 ha), dispersos, uso intensivo de mano de obra, altos costos de producción, mercado inestable y variable, carencia de tecnología apropiada, manejo poscosecha deficiente y debilidad empresarial y gremial (Va1lejo, 2004). La producción de hortalizas en esta zona se caracteriza porque vincula en su mayoría a pequeños y medianos productores que cumplen un papel importante como proveedores de las principales centrales de abasto, no solo de la región, sino de otras zonas del país.

La lechuga es tal vez la más importante hortaliza cultivada en Cundinamarca con un área cultivada de cerca de 636 ha con una producción anual de 9.276 t (Asohofrucol, 2007). En repollo, las zonas más productoras del país son la Sabana de Bogotá, con una área sembrada de 500 ha y una producción anual de 14.475 t anuales, también se incluyen, parte de Cundinamarca y Boyacá, la región del oriente de Antioquia, los alrededores de Pasto (Nariño), la región de Anaime en Tolima, y los alrededores de Manizales en Caldas (Semillas Arroyave, 2006; Asohofrucol, 2007).

Los departamentos más productores de brócoli son Cundinamarca (861 ha), seguido por Boyacá
(146 ha), Nariño (116 ha) y Antioquia (80 ha), con productividades promedios de 11, 21, 6 y 17,4 t ha- 1 , respectivamente, con diferentes materiales híbridos entre los cuales se destacan Legacy, Marathon, Centenario F1 y Centauro F1, entre otras (Asohofrucol, 2007). En apio se reporta un área plantada de 90 ha con un volumen de producción de 1.708 t, distribuidas en los departamentos de Cundinamarca, Antioquia y Boyacá principalmente, siendo uno de los cultivos con mayores tasas de crecimiento entre 2000 y 2006 en área cosechada (23\%) (Asohofrucol, 2007).

Los metales pesados están presentes naturalmente en los suelos, pero en los últimos años se ha presentado una acumulación antropogénica por las actividades industriales, agrícolas y la disposición de residuos de todo tipo (Giuffré et al., 2005). Se considera metal pesado aquel elemento que tiene una densidad igual o superior a $5 \mathrm{~g} \mathrm{~cm}^{3}$ cuando está en forma elemental, o cuyo número atómico es superior a 20 (excluyendo los metales alcalinos y alcalino-térreos) (García y Dorronsoro, 2005), aunque en esta definición, encajan también elementos esenciales para las plantas como $\mathrm{Fe}, \mathrm{Cu}, \mathrm{Mn}, \mathrm{Zn}$ u otros esenciales para los animales como Co, Cr o Ni (Mendoza, 2006). Los elementos As y Se, se consideran como metaloides pero por su efecto contaminante se incluyen dentro de los metales pesados.

Los metales pesados más comunes y ampliamente distribuidos como contaminantes ambientales incluyen plomo $(\mathrm{Pb})$, cadmio $(\mathrm{Cd})$, mercurio (Hg) y el metaloide arsénico (As) (Reilly, 2002). Sin embargo, los metales pesados en las plantas, como en otros seres vivos, son esenciales, ya que son componentes estructurales o catalizadores de los procesos bioquímicos de los organismos, las actividades humanas vierten sobre los recursos de suelo y agua grandes cantidades de esos elementos, generando excesos por acumulación de $\mathrm{Cd}, \mathrm{Hg}, \mathrm{Ni}$ y $\mathrm{Pb}$, entre otros, afectando así las relaciones de las plantas y otros organismos, lo cual origina toxicidades en los ecosistemas (Montenegro, 2002). En general, estos metales 
no son biodegradables y, por consiguiente, pueden acumularse en órganos vitales del cuerpo humano, produciendo efectos tóxicos progresivos (Queirolo et al., 2000). La mayoría de los metales pesados entran en la cadena alimentaria principalmente a través de los cultivos que absorben el agua de riego contaminada.

Las plantas han desarrollado mecanismos altamente específicos para absorber, translocar y acumular sustancias (Lasat, 2000); sin embargo, algunos metales y metaloides no esenciales para los vegetales son absorbidos, translocados y acumulados en la planta debido a que presentan un comportamiento electroquímico similar a los elementos nutritivos requeridos. La fitotoxicidad de los metales pesados se manifiesta particularmente en los suelos ácidos y afecta al crecimiento y la formación de raíces laterales y secundarias (Tadeo y Gómez-Cadenas, 2008).

El objetivo de este estudio fue determinar las concentraciones de metales pesados contaminantes $\mathrm{Pb}, \mathrm{Cd}$, As y $\mathrm{Hg}$, presentes en los suelos y el tejido foliar de los cultivos de lechuga, apio, repollo y brócoli, regados con agua proveniente del río Bogotá en el distrito de riego La Ramada.

\section{MATERIALES Y MÉTODOS}

El material vegetal estuvo compuesto por la lechuga 'Coolguard', el repollo híbrido Delus, el brócoli híbrido Legacy (los tres de marca Seminis de Semillas Arroyave, Bogotá) y el apio 'Tall Utah', marca Seminis de Semillas Saenz, Bogotá.

El experimento, que se llevó a cabo entre los meses de julio y noviembre de 2006, se estableció en lotes comerciales en una finca del municipio de Mosquera, Cundinamarca, vereda San Francisco $\left(4^{\circ} 42^{\prime} \mathrm{N}\right.$ y $\left.74^{\circ} 13^{\prime} \mathrm{W}\right)$ y en una del municipio de Soacha, Cundinamarca, vereda Canoas ( $4^{\circ} 30^{\prime} \mathrm{N}$ y $\left.74^{\circ} 13^{\prime} \mathrm{W}\right)$. El clima en esta zona está caracterizado por una temperatura media anual de $12,6^{\circ} \mathrm{C}$, una precipitación de $670 \mathrm{~mm}$ año-1 y unas $4,2 \mathrm{~h}$ de brillo solar por día.
Para cada uno de los cultivos se demarcó un área experimental consistente en tres franjas de $30 \mathrm{~m}$ de largo por 1,4 $\mathrm{m}$ de ancho, en un diseño de bloques completos al azar, con tres repeticiones. Estas parcelas se ubicaron hacia el centro de los cultivos para asegurar un riego más uniforme y para disminuir efectos de borde.

En cada muestreo se tomaron muestras de suelo, colectado según el modelo de muestreo planteado por Osorio y Ruíz (2005), entre 0 y $20 \mathrm{~cm}$ de profundidad, de las parcelas correspondientes a cada uno de los cultivos y sus repeticiones. De acuerdo con la topografía de los lotes, se realizó un método de muestreo en equis sobre las áreas de los lotes de cada cultivo (Gómez „2006). Las muestras de agua, se colectaron directamente de los aspersores de riego, $1 \mathrm{~L}$ por muestra, y fueron llevadas al Laboratorio de Suelos y Aguas de la Facultad de Agronomía, Universidad Nacional de Colombia, Bogotá, para las determinaciones correspondientes.

El muestreo del suelo en los ensayos correspondió: pH 5,59; CO 4,08\%; N 0,35\%; Ca 10,5, K $0,46, \mathrm{Mg} 3,51, \mathrm{Na} 4,94$ y CIC $27,8 \mathrm{meq} / 100 \mathrm{~g}$, respectivamente; $\mathrm{P} 48,1, \mathrm{Cu} 1,04, \mathrm{Fe} 4,26, \mathrm{Mn}$ 1,49, Zn 21,8 y B 0,94 mg kg-1, respectivamente; Ar $17 \%$, L $47 \%$ y A $36 \%$, con una textura Fr.

Los días de muestreo de material vegetal se realizaron, dependiendo del crecimiento de cada especie, para la lechuga y el apio a los 30, 68 y $103 \mathrm{~d}$ después del transplante (ddt), y para el repollo y brócoli a los 85, 105 y 120 ddt. El muestreo consistió en tomar 15 plantas al azar, en las etapas iniciales del desarrollo del cultivo, disminuyendo la cantidad a medida que estas iban creciendo, esto con el fin de reunir el peso requerido de la muestra para cada uno de los análisis realizados. Las muestras (sin raíz) se secaron en estufas con aire forzado por $48 \mathrm{~h}$ a $70^{\circ} \mathrm{C}$. Aproximadamente $150 \mathrm{~g}$ por muestra se pasaron por un molino eléctrico con un tamiz de $2 \mathrm{~mm}$ y fueron almacenadas para su posterior análisis. Las muestras procesadas de material vegetal, del agua fresca y del suelo fueron enviadas al laboratorio de In- 
geniería Ambiental de la Universidad Nacional de Colombia, Bogotá, para el análisis de metales pesados.

La determinación de los metales pesados correspondió a lo recomendado por la Association of Official Analytical Chemists (AOAC), usando la espectrofotometría de absorción atómica: para el $\mathrm{Cd}$ el método 973.34; para el $\mathrm{Pb}$ el método 972.25 (absorción de llama, análisis tipo III) (FAO, 2007); para el As el método 952.13 (colorimetría, dietilditiocarbamato, análisis tipo II) y para el Hg el método 972.15 (empleando el espectrofotómetro de absorción atómica marca Varian Spectra AA 220 FS, equipado con generador de hidruros) (FAO, 2007). Para el análisis estadístico de los datos, en cada muestreo se tomaron tres repeticiones de cada muestra.

\section{RESULTADOS Y DISCUSIÓN}

\section{Metales pesados en aguas de riego}

No se presentaron diferencias en el nivel de los metales pesados en el agua según los días del muestreo (tabla 1). Siendo los niveles para el uso agrícola en Colombia admisibles hasta $5 \mathrm{mg} \mathrm{L}^{-1}$ para $\mathrm{Pb}$; 0,01 $\mathrm{mg} \mathrm{L}^{-1}$ para $\mathrm{Cd}$ y $0,1 \mathrm{mg} \mathrm{L}^{-1}$ para As (Ministerio de Agricultura, 1984), el Cd se encuentra en el límite permitido para el riego de los cultivos con el agua del distrito La Ramada. Al comparar este resultado (tabla 1) con lo reportado por la CAR (2006) sobre los niveles de $\mathrm{Pb}$ y Cd en la cuenca media del río Bogotá, estos resultados superaron los valores de 2006, cuando se determinaron niveles de $0,032 \mathrm{mg} \mathrm{L}^{-1} \mathrm{~Pb}$ y 0,003 $\mathrm{mg} \mathrm{L}^{-1} \mathrm{Cd}$.
El resultado del presente estudio coincide con lo reportado por Montenegro (2002) sobre la presencia de importantes concentraciones de elementos como $\mathrm{Pb}, \mathrm{Cd}, \mathrm{Cr}, \mathrm{Cu}, \mathrm{Fe}, \mathrm{Ni}$ y $\mathrm{Zn}$ en los ríos Juan Amarillo, Tunjuelo y Fucha que son los principales afluentes del río Bogotá.

El agua puede ser contaminada por metales de manera natural o a través de diferentes procesos de captación, tratamiento, almacenamiento y distribución, afectando su calidad en el punto final de consumo. La situación actual del Distrito con el uso de estas aguas correspondería al tramo de contaminación creciente a alta del río, que va desde el Salitre hasta Tocaima.

Montenegro (2002) informa que el río Bogotá vertía diariamente en su desembocadura al Magdalena $79 \mathrm{~kg}$ de $\mathrm{Pb}, 70 \mathrm{~kg}$ de $\mathrm{Cr}, 20 \mathrm{t}$ de Fe, $5 \mathrm{t}$ de detergentes y 1.473 t de sólidos en suspensión causando un proceso de contaminación difusa. A pesar de que en los muestreos realizados los valores no superaron valores referenciales, sí existe presencia de metales pesados que inciden en la baja calidad del agua del río Bogotá, situación que genera conflictos con los usuarios actuales y los potenciales, en especial para riego en el sur y en el suroccidente de la sabana, donde se encuentra el más importante distrito de riego La Ramada, y los acueductos en la cuenca media y baja (Pérez, 2000).

\section{Metales pesados en suelo}

Los resultados muestran para $\mathrm{Pb}, \mathrm{Cd}$ y As concentraciones cercanas a los rangos normales, determinados por Bowie y Thornton (1985). Estos autores establecieron valores de concentración de

Tabla 1. Concentraciones de metales pesados en agua para riego. Río Bogotá, distrito La Ramada.

\begin{tabular}{|c|c|c|c|c|}
\hline Días de muestreo & $\begin{array}{c}\text { Plomo } \\
\left(\mathrm{mg} \mathrm{L}^{-1}\right)\end{array}$ & $\begin{array}{c}\text { Cadmio } \\
\left(\mathrm{mg} \mathrm{L}^{-1}\right)\end{array}$ & $\begin{array}{c}\text { Arsénico } \\
\left(\mathrm{mg} \mathrm{L}^{-1}\right)\end{array}$ & $\begin{array}{c}\text { Mercurio } \\
\left(\mathrm{mg} \mathrm{L}^{-1}\right)\end{array}$ \\
\hline 30 & $<0,045$ & $<0,01$ & $<0,003$ & $<0,0003$ \\
\hline 68 & $<0,045$ & $<0,01$ & $<0,003$ & $<0,0003$ \\
\hline 103 & $<0,045$ & $<0,01$ & $<0,003$ & $<0,0003$ \\
\hline
\end{tabular}


metales pesados, para $\mathrm{Pb}$ entre 10 y $150 \mathrm{mg} \mathrm{kg}^{-1}$; para Cd entre 1 y $2 \mathrm{mg} \mathrm{kg}^{-1}$ y para As entre 5 y 40 $\mathrm{mg} \mathrm{kg}^{-1}$, válidos para suelos considerados "normales". Por otro lado, las concentraciones encontradas no superan los máximos aceptables en suelos agrícolas de los países de la Unión Europea, reportados por García-Navarro (2005) con $300 \mathrm{mg} \mathrm{kg}^{-1}$ suelo seco para $\mathrm{Pb}, 3 \mathrm{mg} \mathrm{kg}^{-1}$ para $\mathrm{Cd}, 20-50 \mathrm{mg}$ $\mathrm{kg}^{-1}$ para As y $1,5 \mathrm{mg} \mathrm{kg}^{-1}$ para $\mathrm{Hg}$. Sin embargo, los resultados mostraron incrementos considerables con respecto a los determinados por la CAR (2006) en diversos sectores de la cuenca del río Bogotá. Para $\mathrm{Pb}$, nuestros valores estarían separados estos valores de los propuestos por Davies (1977) que fueron de $70 \mathrm{mg} \mathrm{kg}^{-1}$ para un suelo normal. La acidez creciente del suelo puede aumentar la solubilidad del $\mathrm{Pb}$, pero su movilización en el caso del suelo empleado en nuestro trabajo pudo deberse a que la materia orgánica del suelo favorece su acumulación, como lo mencionan Kabata y Pendias (2000). La concentración media de $\mathrm{Hg}$ para los suelos del mundo, raramente excede los $4 \mu \mathrm{g} \mathrm{kg}^{-1}$ asociándose principalmente a suelos orgánicos Kabata-Pendias (2000).

González y Mejía (1995) encontraron en seis zonas hortícolas diferentes en la Sabana de Bogotá, regadas con el agua del río Bogotá, niveles de $\mathrm{Cd}$ entre 0,95 y $2,00 \mathrm{mg} \mathrm{kg}^{-1}$, en una profundidad entre 0 y $30 \mathrm{~cm}$ del suelo, que son muy similares al presente estudio, mientras entre 30 y $60 \mathrm{~cm}$ de profundidad las concentraciones de $\mathrm{Cd}$ fueron solamente entre 0,65 y $1,40 \mathrm{mg} \mathrm{kg}^{-1}$, lo que muestra la mayor acumulación en la parte superficial del suelo y el peligro para las plantas que enraízan solamente en esta capa. Esta misma tendencia la encontraron González y Mejía (1995) para
As con valores entre 6,8 y $12,5 \mathrm{mg} \mathrm{kg}^{-1}$ para la zona entre 0 y $30 \mathrm{~cm}$ del suelo y entre 5,8 y 9,0 $\mathrm{mg} \mathrm{kg}^{-1}$ As en 30 a $60 \mathrm{~cm}$. Bergmann (1993) reporta para el As una concentración máxima 20 $\mathrm{mg} \mathrm{kg}^{-1}$ como tolerable en suelos agrícolas y que una buena aireación del suelo disminuye la adsorción de este elemento por la planta.

Las condiciones de texturas francas de los suelos de la zona de estudio, posiblemente favorecieron la acumulación de estos metales hasta este grado. En forma general, los metales pesados que ingresan en pequeñas cantidades a los suelos se acumulan en lugares específicos de adsorción donde son retenidos fuertemente en los coloides orgánicos e inorgánicos del suelo (Soto, 2006). Los metales pesados cuando se acumulan en concentraciones excesivas son tóxicos para la mayoría de los organismos y frecuentemente se han reportado efectos tóxicos en los microorganismos del suelo (Giller et al., 1998), especialmente en suelos poco estructurados y con baja materia orgánica (Acevedo et al., 2005), como fue encontrada en la zona del estudio con 4,08\% carbono orgánico.

García-Navarro (2005) comenta que solo se puede hablar de contaminación por metales pesados cuando el contenido en los mismos excede considerablemente de los valores habituales en el tipo de suelo que se está considerando, situación no observada en el presente trabajo. Los materiales constitutivos de los suelos como limos y arcillas fueron determinantes en la acumulación y disponibilidad de estos elementos (Soto, 2006).

De acuerdo con Vargas y Mejía (1996) el Hgy el Pb pueden presentarse en una o más de las siguientes

\section{Tabla 2. Concentración de metales pesados determinados en suelo cultivado con las cuatro hortalizas en Soacha,} Cundinamarca.

\begin{tabular}{|c|c|c|c|c|}
\hline Muestre0 & $\begin{array}{c}\text { Plomo } \\
\left(\mathrm{mg} \mathrm{kg}^{-1}\right)\end{array}$ & $\begin{array}{c}\text { Cadmio } \\
(\mathrm{mg} \mathrm{kg}\end{array}$ & $\begin{array}{c}\text { Arsénico } \\
\left(\mathrm{mg} \mathrm{kg}^{-1}\right)\end{array}$ & $\begin{array}{c}\text { Mercurio } \\
\left(\mathrm{mg} \mathrm{kg}^{-1}\right)\end{array}$ \\
\hline $1(30 \mathrm{ddt})$ & $11,08 \mathrm{a}$ & $0,86 \mathrm{~b}$ & $1,42 \mathrm{~b}$ & $<0,03$ (nd) \\
\hline $2(68 \mathrm{ddt})$ & $9,53 \mathrm{~b}$ & $0,68 \mathrm{c}$ & $1,35 \mathrm{c}$ & $<0,03$ (nd) \\
\hline $3(103 \mathrm{ddt})$ & $11,83 \mathrm{a}$ & $1,73 \mathrm{a}$ & $1,75 \mathrm{a}$ & $<0,03$ (nd) \\
\hline
\end{tabular}

Promedios con letras distintas indican diferencia significativa según la prueba de Tukey $(P \leq 0,05)$; ddt, días después de transplante; nd, niveles no detectables. 
formas químicas: (a) disueltos en la solución del suelo; (b) en forma intercambiable; (c) ligados a compuestos orgánicos; (d) ocluidos en óxidos de $\mathrm{Fe}, \mathrm{Al}, \mathrm{Mn}$, etc.; (e) formando compuestos definidos, o (f) ligados a la fracción arcilla.

Es importante tener en cuenta que la determinación de los metales pesados totales en el suelo es una medida útil para estimar la contaminación del suelo, pero no informa sobre su solubilidad o disponibilidad para las plantas (Walter et al., 2003).

\section{Metales pesados en tejido foliar}

Los contenidos de metales pesados en el tejido foliar están ligados a los momentos fisiológicos en los que se llevaron a cabo los muestreos, ya que las plantas tienen diversas rutas metabólicas (Miranda et al., 2008). En esto como en otro estudio relacionado (Ni et al., 2002) no se encontraron síntomas de deficiencia nutricional o de toxicidad visibles en las especies investigadas.

\section{Lechuga y apio}

La tabla 3 muestra el comportamiento de los cuatro metales pesados en la parte comestible de las dos hortalizas, observando niveles mayores en la lechuga.

El $\mathrm{Pb}$ presentó concentraciones altas en las hojas de lechuga a los $74 \mathrm{ddt}$ con $0,20 \mathrm{mg} \mathrm{kg}^{-1} \mathrm{PF}$ (tabla
3), que se acercan al valor máximo permitido de 0,30 $\mathrm{mg} \mathrm{kg}^{-1} \mathrm{PF}$ para hortalizas de hoja, según la Unión Europea (2006). Se debe resaltar que los valores de $\mathrm{Pb}$, para las dos hortalizas superan el límite para alimentos infantiles para lactantes y niños de corta edad de la Unión Europea (2008) que tienen un máximo de $0,02 \mathrm{mg} \mathrm{kg}^{-1} \mathrm{PF}$.

Marschner (2002) afirma que la lechuga es una planta capaz de absorber grandes cantidades de $\mathrm{Pb}$ de suelos que se encuentren altamente contaminados. Sobre el efecto del $\mathrm{Pb}$ en los cultivos se han demostrado que valores altos reducen drásticamente el crecimiento, máxime si hay deficiencia de fosfato (Bergmann, 1993), situación que no se evidenció en el presente estudio, donde los contenidos de $\mathrm{Pb}$ determinados variaron entre 4,2 y $6 \mathrm{mg} \mathrm{L}^{-1}$, durante los cuatro muestreos realizados. Montenegro (2000) afirma que la concentración total del Cd en el suelo (fracciones móviles más inmóviles), al igual que la tasa de difusión del elemento móvil a través de la solución del suelo, incide en la cantidad absorbida por las plantas y de estas, es la fracción móvil la que constituye el verdadero riesgo de toxicidad para las plantas. Las concentraciones de $\mathrm{Cd}$ en la lechuga y el apio, con 0,40 y $0,43 \mathrm{mg} \mathrm{kg}^{-1} \mathrm{PF}$, a los $74 \mathrm{ddt}$, superan ampliamente lo establecido por la Unión Europea (2006) con 0,20 $\mathrm{mg} \mathrm{kg}^{-1} \mathrm{PF}$.

Este comportamiento se puede relacionar con varios factores. De acuerdo con las condiciones agroecológicas, un suelo poco encharcado pudo

Tabla 3. Concentración de metales pesados, determinados en tejido foliar fresco (PF) de lechuga.

\begin{tabular}{|c|c|c|c|c|}
\hline Muestreo & $\begin{array}{c}\text { Plomo } \\
\left(\mathrm{mg} \mathrm{kg}^{-1}\right)\end{array}$ & $\begin{array}{l}\text { Cadmio } \\
\left(\mathrm{mg} \mathrm{kg}^{-1}\right)\end{array}$ & $\begin{array}{l}\text { Arsénico } \\
\left(\mathrm{mg} \mathrm{kg}^{-1}\right)\end{array}$ & $\begin{array}{l}\text { Mercurio } \\
\left(\mathrm{mg} \mathrm{kg}^{-1}\right)\end{array}$ \\
\hline \multicolumn{5}{|c|}{ Lechuga } \\
\hline 1 (30 ddt) & $<0,06$ (nd) & $<0,02$ (nd) & $0,09 \mathrm{~b}$ & $0,07 \mathrm{a}$ \\
\hline 2 (74 ddt) & 0,20 & $0,40 \mathrm{a}$ & $0,19 a$ & $0,06 \mathrm{a}$ \\
\hline 3 (89 ddt) & $<0,06$ (nd) & $0,04 \mathrm{~b}$ & $0,02 \mathrm{c}$ & $0,01 \mathrm{~b}$ \\
\hline \multicolumn{5}{|c|}{ Apio } \\
\hline 1 (30 ddt) & $<0,04$ (nd) & $0,009 \mathrm{~b}$ & $0,02 a$ & $<0,003$ (nd) \\
\hline 2 (74 ddt) & $<0,04$ (nd) & $0,430 \mathrm{a}$ & $0,02 \mathrm{a}$ & $<0,002$ (nd) \\
\hline 3 (89 ddt) & $<0,04$ (nd) & $0,008 \mathrm{~b}$ & $0,02 \mathrm{a}$ & 0,020 \\
\hline
\end{tabular}

Promedios con letras distintas indican diferencia significativa según la prueba de Tukey $(P \leq 0,05)$; ddt, días después de transplante; nd, niveles no detectables. 
ocasionar una mayor absorción de Cd (Acevedo et al., 2005); sumado a esto, la cercanía de los lotes de muestreo a las factorías que se ubican alrededor del sitio experimental, que favorecen la contaminación aérea, la cual puede influir la concentración en la planta (Bergmann, 1993) en esta fase del cultivo $y$, posiblemente, la dificultad para retirar la capa contaminante adherida a las hojas muestreadas.

Otros factores que pudieron influenciar el incremento de $\mathrm{Cd}$ se relacionan con las diferencias en la morfología de las raíces, teniendo la lechuga numerosas raíces delgadas que pueden acumular más metales que otras plantas con raíces más gruesas y suculentas (Schierup y Larsen, 1981). Además, se ha comprobado que raíces de la lechuga translocan hacía los brotes la mayor cantidad del Cd absorbido comparado con otras especies (Jarvis et al., 1976). De igual manera, factores de muestreo que pudieron haber influenciado la respuesta tienen que ver con el uso de hojas más viejas (externas) de la planta que se caracterizan por su mayor capacidad de acumular metales pesados que las hojas jóvenes (internas) (Das et al., 1997), debido a la presencia de péptidos que ligan estos metales (Vogeli-Lange y Wagner, 1990).

El Cd es uno de los metales traza del suelo más solubles (Jansson, 2002), peligroso debido a su alta movilidad inhibe drásticamente el crecimiento celular y de toda la planta en concentraciones tóxicas (Prasad, 1995). Mengel et al. (2001) reportan que la tasa de absorción del Cd por la lechuga es particularmente alta, comparado con otras especies. Estudios realizados por Insuasty et al. (2008), evaluando la incorporación de dosis crecientes de $\mathrm{Cd}$ al cultivo de papa, mostraron que la acumulación de este metal en la planta es mayor en las concentraciones altas de este elemento en el suelo y que se acumula en mayor porcentaje en los primeros $15 \mathrm{~cm}$ de este, lo que manifiesta el potencial de toxicidad para los cultivos de porte bajo con un sistema de raíces superficial.
Los síntomas más generales de toxicidad por Cd son atrofia y clorosis, esta puede aparecer debido a una interacción directa o indirecta con el Fe, el Zn, el P y el Mn. Das et al. (1998) reportan que aun cuando los efectos del Cd varían a nivel de especie, e incluso varietal, en general el Cd interfiere en la absorción y transporte de varios elementos (Ca, Mg, P y K) y del agua.

González y Mejía (1995) regaron lechugas en bolsas de PE (2 L) con agua contaminada por Cd en $0,1 \mathrm{mg} \mathrm{L}^{-1}$ (que corresponde a una concentración 10 veces mayor que en el estudio presente) lo que aumentó el nivel de Cd en el tejido foliar a 6,12 $\mathrm{mg} \mathrm{kg}^{-1} \mathrm{PS}$, concentración muy superior comparada con el límite internacional permitido.

Por otro lado, McLaughlin et al. (1999) reportan que la acumulación del Cd en las hortalizas puede ser más alta en suelos salinos, que es el caso de los terrenos en la zona de influencia del río Bogotá (Carranza et al., 2009), debido a que la formación de complejos entre el Cl y el Cd aumenta la movilidad y la absorción de este por las plantas.

Para As y Hg la Unión Europea (2006) no establece niveles tóxicos en hortalizas, solamente para el $\mathrm{Hg}$ los ha fijado en $0,5-1,0 \mathrm{mg} \mathrm{kg}^{-1}$ en diferentes tipos de pescado.

La absorción de Hg por la lechuga fue menor que la de los otros metales pesados estudiados, debido, muy posiblemente, por la menor concentración en agua (tabla 1) y suelo (tabla 2) y la fuerte adsorción de los iones Hg en la sustancia orgánica del suelo (Bergmann, 1993). También, es de considerar que la absorción máxima de $\mathrm{Hg}^{2+}$ ocurre en rangos de $\mathrm{pH}$ entre 4,0 y 5,0 (McLaughlin et al., 1999), que fue mayor en el presente estudio $y$, además, la absorción de $\mathrm{Hg}$ se disminuye significativamente con la presencia de $\mathrm{Cl}$ (Hogg et al., 1978), situación que está dada por la salinidad del agua y del suelo en la zona de influencia del río Bogotá (Carranza et al., 2009). 


\section{Repollo y brócoli}

En general, la acumulación de los metales $\mathrm{Pb}$ y $\mathrm{Cd}$ en el tejido comestible de repollo y brócoli (tabla 4) fue menor que en el de la lechuga (tabla 3), teniendo en cuenta que esta última es un bioacumulador de dichos metales (Mengel et al., 2001). También Bergmann (1993) clasifica la acumulación relativa del repollo para metales pesados como baja a mediana comparada con la lechuga que es alta. No obstante, para el caso del $\mathrm{Hg}$ el repollo pasó en la última medición (120 ddt) los niveles encontrados en lechuga.

Para el $\mathrm{Pb}$ los valores detectados en el tejido foliar fueron $<0,05 \mathrm{mg} \mathrm{kg}^{-1}$ e inferiores a los niveles de detección exacta del equipo (tabla 4) y así por debajo de la norma de la Unión Europea (2007) para hortalizas del género Brassica con $0,3 \mathrm{mg} \mathrm{kg}$ ${ }^{1} \mathrm{PF}$. Teniendo en cuenta que para la elaboración de alimentos infantiles para lactantes y niños de corta edad rigen normas más estrictas, este grupo de población no debería consumir repollo ni brócoli contaminados porque pasan los 0,02 mg kg-1 de $\mathrm{Pb}$ en el alimento preparado (Unión Europea, 2008). En Colombia, la NTC 1474 establece los límites máximos de contaminantes para lactantes y niños de corta edad para el $\mathrm{Pb}$ (límite máximo 0,02 $\mathrm{mg} \mathrm{kg}^{-1} \mathrm{PF}$ ), determinado de acuerdo con lo indicado en la norma AOAC 999.11 (Icontec, 2009).
El Cd en la parte comestible de repollo y brócoli no pasó los niveles máximos establecidos por la Unión Europea (2006) con 0,20 mg kg-1 PF (tabla 4). Verma et al. (2007) modelaron la toma de Cd por la planta de repollo y encontraron un nivel notablemente menor que en zanahoria (máxima absorción) y espinaca (mediana absorción), mientras la toma de $\mathrm{Cd}$ por el rábano fue mínimo.

Los niveles de As en la localidad de Soacha fueron mayores que los contenidos medios reportados por Kabata-Pendias (2000) para repollo con 0,02$0,05 \mathrm{mg} \mathrm{kg}^{-1}$ peso seco (PS), los cuales estuvieron entre 0,48 y $0,57 \mathrm{mg} \mathrm{kg}^{-1}$ (PS) (que correspondió a 0,04-0,06 mg kg-1 PF en la tabla 4). Resultado que muestra las concentraciones altas de este metaloide en el repollo en la zona de estudio comparado con el nivel promedio internacional.

El Hg encontrado en el tejido comestible de repollo y brócoli (tabla 4) fue menor que en lechuga (tabla 3), solamente el tercer muestreo en repollo (tabla 4) superó a las demás mediciones de este metal, que se podría ver como un efecto de acumulación de Hg en la planta que también se presentó en apio, pero no en brócoli y lechuga (tabla 3). Como se mencionó para el caso de la lechuga, por un exceso de $\mathrm{Cl}^{-}$la absorción de $\mathrm{Hg}^{+}$en las partículas minerales del suelo y la materia orgánica disminuye debido a la formación de com-

Tabla 4. Concentración de metales pesados determinados en tejido foliar fresco (PF) de repollo y brócoli.

\begin{tabular}{|c|c|c|c|c|}
\hline Muestreo & $\begin{array}{c}\text { Plomo } \\
\left(\mathrm{mg} \mathrm{kg}^{-1}\right)\end{array}$ & $\begin{array}{l}\text { Cadmio } \\
\left(\mathrm{mg} \mathrm{kg}^{-1}\right)\end{array}$ & $\begin{array}{l}\text { Arsénico } \\
\left(\mathrm{mg} \mathrm{kg}^{-1}\right)\end{array}$ & $\begin{array}{l}\text { Mercurio } \\
\left(\mathrm{mg} \mathrm{kg}^{-1}\right)\end{array}$ \\
\hline \multicolumn{5}{|c|}{ Repollo } \\
\hline 1 (85 ddt) & $<0,05$ (nd) & $<0,010$ (nd) & $0,06 \mathrm{a}$ & $<0,003$ (nd) \\
\hline 2 (105 ddt) & $<0,04$ (nd) & $<0,008$ (nd) & $0,04 \mathrm{a}$ & $<0,002$ (nd) \\
\hline 3 (120 ddt) & $<0,04$ (nd) & $<0,008$ (nd) & $0,05 \mathrm{a}$ & 0,150 \\
\hline \multicolumn{5}{|c|}{ Brócoli } \\
\hline 1 (85 ddt) & $<0,05$ (nd) & $<0,01$ (nd) & $0,05 \mathrm{a}$ & $<0,003$ (nd) \\
\hline 2 (105 ddt) & $<0,06$ (nd) & $<0,01$ (nd) & $0,05 a$ & $<0,004$ (nd) \\
\hline 3 (120 ddt) & $<0,05$ (nd) & $<0,01$ (nd) & $0,05 \mathrm{a}$ & 0,004 (nd) \\
\hline
\end{tabular}

Promedios con letras distintas indican diferencia significativa según la prueba de Tukey $(P \leq 0,05)$; ddt, días después transplante; nd, niveles no detectables. 
plejos de $\mathrm{Hg}-\mathrm{Cl}$ altamente estables que son poco adsorbidos (Acevedo et al., 2005). Por lo general, la absorción de $\mathrm{Hg}$ desde el suelo a las plantas es baja, constituyéndose en una barrera para la traslocación desde las raíces a las partes aéreas de las plantas (Patra y Sharma, 2000), en consecuencia la cadena alimenticia no se ve tan afectada como en el caso del Cd (Bergmann, 1993).

\section{CONCLUSIONES}

- Se observó en el agua de riego, proveniente del río Bogotá, que las concentraciones de metales pesados, especialmente el cadmio, están cercanas a los límites establecidos por las normas vigentes nacionales e internacionales. Los resultados mostraron incrementos considerables con respecto a los determinados anteriormente por la CAR en diversos sectores de la cuenca del río Bogotá.
- En el suelo de la plantación hortícola no se encontraron niveles tóxicos de metales pesados estudiados.

- A los $74 \mathrm{ddt}$, se presentaron niveles tóxicos del cadmio en la lechuga y el apio que sobrepasan el nivel máximo permitido por la Unión Europea.

- No se encontró contaminación excesiva por plomo, arsénico y mercurio en las hortalizas investigadas.

- Sin embargo, las concentraciones de plomo superaron los niveles permitidos en las cuatro hortalizas, para ser usadas en alimentos infantiles.

- Los suelos hortícolas urbanos y periurbanos deben ser cuidadosamente monitoreados considerando el riesgo de entrada de metales en la cadena alimentaria.

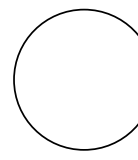

AGRADECIMIENTOS

Los autores agradecen a los expertos de los laboratorios de suelos y aguas de la Facultad de Agronomía y al personal técnico del Instituto de Ensayos e Investigaciones (IEI) de la Universidad Nacional de Colombia por su apoyo en la ejecución de esta investigación.

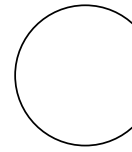

Acevedo, E.; M.A. Carrasco; O. León; E. Martínez; P. Silva; G. Castillo; I. Ahumada; G. Borie y S. González. 2005. Criterios de calidad de suelo agrícola. Gobierno de Chile, Ministerio de Agricultura (SAG), Santiago.

Asohofrucol. 2007. Apio, brócoli, lechuga y repollo. En: http://frutasyhortalizas.com.co; consulta: diciembre de 2007

Bergmann, W. 1993. Ernährungsstörungen bei Kulturpflanzen. Gustav Fischer Verlag, Jena, Alemania.

Bowie, S.H.U. e I. Thornton (eds.). 1985. Environmental geochemistry and health. D. Reidel Publishing Company, Lancaster, PA.

\section{REFERENCIAS BIBLIOGRÁFICAS}

CAR. 1991. Programa de aforo y muestreo de los ríos Bogotá, Ubaté y Suárez y sus afluentes; muestreo a embalses, lagos y lagunas, preservación y transporte de muestras. Informe final. Ingeseries Ltda., Bogotá.

CAR. 2006. Objetivos de calidad del agua para la cuenca del río Bogotá a lograr en el año 2020. Acuerdo No. 43 del 17 de octubre de 2006. Corporación Autónoma Regional de Cundinamarca, Bogotá.

Carranza, C.; O. Lanchero; D. Miranda y B. Chaves. 2009. Análisis de crecimiento de lechuga (Lactuca sativa L.) 'Batavia' cultivada en un suelo salino de la Sabana de Bogotá. Agron. Colomb. 27(1), 4148. 
CONPES. 2004. Estrategia para el manejo ambiental del Río Bogotá. Documento Conpes 3320. Consejo Nacional de Política Económica y Social. Departamento Nacional de Planeación. Ministerio de Ambiente, Vivienda y Desarrollo Territorial, Bogotá.

CPT. 1985. Estudio para la ampliación del Distrito de Riego La Ramada. Anexo 2-Agrología. Corporación Autónoma Regional de Cundinamarca (CAR), Bogotá.

Das, P.; S. Smarantay y G.R. Rout. 1998. Studies in Cd toxicity in plants: A review. Environ. Pollution 98, 29-36.

EAAB. 2002. Estudio sobre la calidad del agua del río Bogotá. Empresa de Acueducto y Alcantarillado de Bogotá, Bogotá.

FAO. 2007. Código de prácticas de higiene para las frutas y hortalizas frescas. pp. 162-192. En: Codex alimentarius. Organización Mundial de la Salud. Organización de las Naciones Unidas para la Agricultura y la Alimentación, Roma.

García, I. y C. Dorronsoro. 2005. Contaminación por metales pesados. En: Tecnología de suelos. Departamento de Edafología y Química Agrícola, Universidad de Granada, Granada, España.

García-Navarro, A. 2003. Gestión y conservación del suelo. Lección 4. Contaminación del suelo. Contaminantes específicos. Metales pesados. Departamento de Biología y Producción de los Vegetales, Universidad de Extremadura, Badajoz, España.

Giller, K.E.; E. Witter y S.P. McGrath. 1998. Toxicity of heavy metals to microorganism and microbial processes in agricultural soils: a review. Soil Biol. Biochem. 30(10-11), 1389-1414.

Giuffré, L.; S. Ratto; L. Marbán; J. Schonwald y R. Romaniuk 2005. Riesgo por metales pesados en horticultura urbana. Ciencia del Suelo 23(1), 101-106.

Gómez, M.I. 2006. Manual técnico de fertilización de cultivos. Microfertisa S.A., Produmedios, Bogotá.

González, S. y L. Mejía. 1995. Contaminación con cadmio y arsénico en suelos y hortalizas de un sector de la cuenca del río Bogotá. Suelos Ecuatoriales 25, 51-56.

Hogg, T.J.; K.W.B. Stewart y J.R. Bettany. 1978. Influence of the chemical form of mercury on its adsorption and ability to leach through soils. J. Environ. Qual. 7, 440-445.

Icontec. 2009. Alimentos complementarios para niños lactantes y de corta edad. NTC 1474. Segunda actualización. Instituto Colombiano de Normas Técnicas y Certificación, Bogotá.
Insuasty, L.; H. Burbano y J. Menjivar. 2008. Dinámica del cadmio en suelos cultivados con papa en Nariño, Colombia. Acta Agron. 57(1), 51-54.

Jansson, G. 2002. Cadmium in arable crops. The influence of soil factors and liming. Tesis doctoral. Department of Soil Sciences, Swedish University of Agricultural Sciences, Uppsala, Suecia.

Jarvis, S.C.; L.H.P. Jones y M.J. Hopper. 1976. Cadmium uptake from solution by plants and its transport from roots do shoots. Panta Soil 44, 179-191.

Kabata-Pendias, A. 2000. Trace elements in soils and plants. 3a. ed. CRC Press, Boca Raton, FL.

Lasat, M.M. 2000. The use of plants for the removal of toxic metals from contaminated soil. American Association for the Advancement of Sciencie, Environmental Protection Agency (EPA), Washington DC.

Marschner, H. 2002. Mineral nutrition of higher plants. Academic Press, London.

McLaughlin, M.J., D.R. Parker y J.M. Clarke. 1999. Metals and micronutrients - food safety issues. Field Crops Res. 60, 143-163.

Mendoza. P.M. 2006. Estudio de metales pesados en suelos bajo cultivos hortícolas de la provincia de Castellón. Publicaciones Server. Universidad de Valencia, Valencia, España.

Mengel, K.; E.A. Kirkby; H. Kosegarten y T. Appel. 2001. Principles of plant nutrition. Kluwer Academic Publishers, Dordrecht, The Netherlands.

Ministerio de Agricultura. 1984. Decreto 1594 del 26 de junio de 1984 en cuanto a usos del agua y residuos líquidos. Bogotá.

Miranda L., D., C. Carranza y G. Fischer. 2008. Calidad del agua de riego en la Sabana de Bogotá. Facultad de Agronomía, Universidad Nacional de Colombia, Bogotá.

Montenegro, R.O. 2002. Contaminación química de suelos y cultivos. Estrategias para la productividad de los suelos agrícolas. Sociedad Colombiana de la Ciencia del Suelo, Capitulo Tolima, Bogotá.

Ni, W.Z.; X.X. Long y X.E. Wang. 2002. Studies o the criteria of cadmium pollution in growth media of vegetable crops based on the hygienic limit of cadmium in food. J. Plant Nutr. 25(5), 957-968.

Osorio, N. y O. Ruíz. 2005. Guía para el muestreo de suelos. Escuela de Geociencias, Facultad de Ciencias, Universidad Nacional de Colombia, Medellín, Colombia. 
Patra, M. y A. Sharma. 2000. Mercury toxicity in plants. Bot. Rev. 66, 379-422.

Pérez, A. 2000. La estructura ecológica especial de la Sabana de Bogotá. Sociedad Geográfica de Colombia, Academia de Ciencias Geográficas, Bogotá.

Prasad, M.N.V. 1995. Cadmium toxicity and tolerance in vascular plants. Environ. Exp. Bot. 35(4), 525-545.

Queirolo, F.; S. Stegen; M. Restovic; M. Paz; P. Ostapczuk; M.J. Schwuger y L. Muñoz. 2000. Total arsenic, lead, and cadmium levels in vegetables cultivated at the Andean villages of northern Chile. Sci. Total Environ. 255, 75-84.

Reilly, C. 2002. Metal contamination of food. Its significance for food quality and human health. Blackwell Science Ltd., Oxford, UK.

Schierup, H. y V.J. Larsen. 1981. Macrophyte cycling of $\mathrm{Zn}, \mathrm{Cu}, \mathrm{Pb}$ and $\mathrm{Cd}$ in the littoral zone of a polluted and non-polluted lake. I. Availability, uptake and translocation of heavy metals in Phragmites australis (Cav.). Trin. Aquatic Bot. 11, 179-210.

Semillas Arroyave. 2006. Repollo Delus. En: http:// www.semillasarroyave.com. 2 p.; consulta: diciembre de 2007.

Soto. J.S.H. 2006. Contaminación del río Rimac por metales pesados y efecto en la agricultura en el cono este de Lima metropolitana. Trabajo de grado. Universidad Nacional Agraria La Molina, Lima.

Tadeo, F.R. y A. Gómez-Cadenas. 2008. Fisiología de las plantas y el estrés. pp. 577-597. En: Azcón-Bieto, J. y M. Talón. Fundamentos de fisiología vegetal. $2 \mathrm{a}$ ed. McGraw-Hill Interamericana, Madrid.
Unión Europea. 2006. Contenido máximo de determinantes contaminantes en los productos alimenticios. Reglamento CE 1881/2006 de la Comisión. Diario Oficial de la Unión Europea 20.12.2006, L 364/5-L364/24.

Unión Europea. 2008. Contenidos máximos de determinados contaminantes. En: http://europa.eu/legislation_summaries/food_safety/contamination environmental_factors/121290_es.htm; consulta: noviembre de 2008 .

Vallejo, F.A. 2004. Hortalizas en Colombia. Facultad de Ciencias Agropecuarias, Universidad Nacional de Colombia, Palmira.

Vargas, O. y L. Mejía. 1996. Evaluación de la contaminación por $\mathrm{Hg}$ y $\mathrm{Pb}$ en suelos hortícolas de la cuenca alta del río Bogotá y del efecto del $\mathrm{Hg}$ y $\mathrm{Pb}$ de las aguas de riego y de suelos en varias hortalizas. II Simposio Internacional de Geoquímica Ambiental. Ingeominas, Universidad Nacional de Colombia, Cartagena, Colombia.

Verma, P., K.V. George; H.V. Singh y R.N. Singh. 2007. Modeling cadmium accumulation in radish, carrot, spinach and cabbage. Appl. Math. Model. 31, 1652-1661.

Vogeli-Lange, R. y G.J. Wagner. 1990. Subcellular localization of cadmium-binding peptides in toabacco leaves. Plant Physiol. 92, 1086-1093.

Walter, I.; F. Martínez y G. Cuevas. 2003. Dinámica de los metales pesados en un suelo degradado enmendado con residuos orgánicos urbanos. pp. 367-371 En: Álvarez-Benedí, J. y P. Marinero (eds.). Estudios de la Zona No Saturada del Suelo Vol. VI. Departa- 